\title{
Flaubert, Spencer et le paradigme évolutionniste
}

\section{Gisèle Séginger}

\section{(2) OpenEdition}

\section{Journals}

Édition électronique

URL : http://journals.openedition.org/aes/293

DOI : $10.4000 /$ aes.293

ISSN : 2258-093X

\section{Éditeur}

Laboratoire LISAA

\section{Référence électronique}

Gisèle Séginger, «Flaubert, Spencer et le paradigme évolutionniste », Arts et Savoirs [En ligne], 4 | 2014, mis en ligne le 15 mai 2014, consulté le 20 avril 2019. URL : http://journals.openedition.org/aes/293 ; DOI : 10.4000/aes.293

Ce document a été généré automatiquement le 20 avril 2019.

Centre de recherche LISAA (Littératures SAvoirs et Arts) 


\title{
Flaubert, Spencer et le paradigme évolutionniste
}

\author{
Gisèle Séginger
}

Quand Flaubert a-t-il lu Spencer? Ni la Correspondance ni les manuscrits ne permettent de répondre avec certitude à cette question. Flaubert cite pour la première fois le nom de Spencer dans une lettre de 1878, à un moment où la rédaction de Bouvard et Pécuchet est bien engagée, puisqu'il y travaille depuis 1872. La Correspondance donne peu d'indications. On sait seulement qu'il lit spencer pour préparer un chapitre sur l'éducation, qu'il consulte plusieurs volumes, et qu'il manifeste d'abord un enthousiasme un peu général et vague à l'égard de ce positiviste moins strictement matérialiste que les positivistes français avant d'éprouver une déception à la lecture de son livre sur l'éducation' ${ }^{1}$. Toutefois lorsqu'on lit la Correspondance de Flaubert des années 1850 et les œuvres de Spencer on est frappé par une convergence de pensée. Mon hypothèse pourtant ne sera pas celle d'une lecture précoce qui aurait pu être faite directement dans la langue anglaise, même si cela n'est pas totalement impossible ${ }^{2}$. Je partirai plutôt de l'hypothèse d'une lecture tardive (en 1878) et en définitive peu marquante au-delà d'une sorte de plaisir de reconnaissance qui suscite cet enthousiasme : « Voilà un homme, celuilà $! »^{3}$ Lorsqu'on lit Spencer après avoir lu Flaubert et sa Correspondance, on éprouve l'impression de reconnaitre plus d'idées qu'on ne fait de réelles découvertes, en dehors bien sûr de l'étonnante puissance intégrative du système spencérien que l'on ressent à la lecture des Premiers principes. Or, il est bien évident qu'il est impossible de retourner la question initiale et de s'interroger sur une possible lecture de Flaubert par Spencer 4 ! Mon hypothèse est donc que Spencer actualise sous la forme d'un système philosophique un paradigme dont la construction progressive dans la première moitié du siècle a été possible grâce à la circulation d'éléments d'abord dispersés puis coordonnés et structurés, bien identifiés finalement comme des éléments à rattacher à un paradigme qu'on appelle évolutionniste et à des œuvres fondatrices. Fondatrices non en ce qu'elles feraient brusquement une révolution mais en ce qu'elles modélisent et structurent une nouvelle approche du monde, donnant visibilité et acceptabilité à une conception cohérente qui tend à supplanter dans la communauté scientifique et intellectuelle sinon complètement du moins largement d'anciens modes de pensée. 
2 J'emprunte la notion de paradigme au philosophe et historien des sciences américain, Thomas Kuhn, qui l'a théorisée en 1962 dans La Structure des révolutions scientifiques. Grâce à cette notion, il pense l'histoire des sciences et les révolutions scientifiques dans une dimension collective et structurante par rapport à une communauté scientifique et des institutions académiques et non comme une série cumulative de découvertes et de progrès ou un palmarès de grands scientifiques. Un paradigme - à ne pas confondre simplement avec une conception du monde - implique à la fois des manières de penser, un redécoupage du réel et de nouveaux objets d'étude, des méthodes d'investigation, une forme de réception et d'acceptabilité qui oriente les travaux en cours, une capacité à absorber et retraiter des objections. Il implique des positions, un certain rapport avec les institutions académiques, un impact sur l'enseignement. Il ne se fonde pas sur un ensemble de découvertes, il se définit plutôt par un certain nombre de règles qui régissent l'investigation, par une approche du monde marquée épistémologiquement voire sociologiquement et idéologiquement. Le paradigme donne une nouvelle figurabilité au monde; il oriente les questions que les scientifiques lui adressent, il donne aussi une visibilité à un groupe de savants qui partagent les mêmes perspectives et pratiques. Il s'appuie sur une discipline de référence, sur des modèles de pensée partagés. Le paradigme nous renseigne donc sur une approche commune du réel et des sciences qui en traitent, sur l'origine des règles respectées par une communauté scientifique aux contours plus ou moins définis. Thomas Kuhn théorise cette notion pour expliquer la dynamique des sciences et pour se débarrasser d'une conception convenue et simpliste du progrès 5 .

3 Je me propose d'utiliser pour ma part cette notion dans le domaine de l'histoire des idées. Elle permet de comprendre trois points : le succès de Spencer en France (les lecteurs qui assurent son succès y retrouvent un paradigme), la brièveté de ce succès liée à l'histoire des paradigmes à l'extrême fin du siècle et au tout début du suivant, et le rapport de Flaubert à Spencer, mon hypothèse étant que la pensée de Flaubert dans sa Correspondance et sa pratique du roman entretiennent incontestablement un rapport au paradigme évolutionniste plus qu'un rapport direct avec les textes de Spencer. Pour autant, il ne s'agit pas de se débarrasser de Spencer ou de contourner la question de la réception de Spencer en France, mais bien plutôt de mieux comprendre la place du philosophe anglais dans un travail à l'œuvre dans le siècle, et auquel collabore tout un ensemble de penseurs. C'est cela qui permet d'expliquer l'écho qu'il a trouvé en France. La notion de paradigme présente l'avantage de proposer une conception différente de l'histoire des sciences: elle déplace l'attention des figures géniales, des inventeurs qui polarisent brusquement l'intérêt à certains moments vers une communauté complexe, des réseaux, une circulation d'idées, des phénomènes de coalescence; elle déplace l'intérêt de l'événement vers la moyenne durée, de la découverte vers la structuration d'un groupe actif dans la transformation des manières de penser.

4 Je voudrais surtout montrer le rôle sinon des textes de Spencer du moins d'un paradigme auquel il participe dans la réflexion de Flaubert et dans le projet de Bouvard et Pécuchet, dans la composition d'un roman philosophique qui déconstruit les savoirs, les philosophies, les croyances mais n'en implique pas moins une manière d'aborder le monde. Elle ne se formule pas - impersonnalité oblige - mais elle se devine parce qu'elle agit dans l'organisation du récit et dans la conception d'un débat infini entre deux personnages. Le contact entre Flaubert et Spencer, même lorsque l'écrivain a enfin lu les textes, implique ce rapport au paradigme vers lequel fait signe aussi la philosophie de 
Spencer, mais qui ne se réduit pas à cette philosophie. Avant d'en arriver à la poétique de Bouvard et Pécuchet, j'aurai besoin de faire un détour par les débats impliqués dans la réception de Spencer parce qu'ils sont révélateurs d'oppositions qui structurent le champ discursif de l'époque, qui agissent dans la formation du paradigme évolutionniste, et que Flaubert prend aussi en charge lorsqu'il aborde le projet de Bouvard et Pécuchet à partir de 1872.

Dans sa Correspondance, Flaubert défend une approche positive des faits humains ${ }^{6}$ - sans préjugés moraux ou religieux - et beaucoup moins ce qu'on désigne du nom de positivisme dans le contexte philosophique du XIX ${ }^{e}$ siècle. Encore faut-il préciser que sa position est très différente s'il s'agit du positivisme de Comte qui sacralise la science - on sait que Flaubert y trouve des "Californies de rire $»^{7}-$ ou des positivistes de la seconde génération (Littré, Taine, Baudry, Maury, Berthelot, Claude Bernard) dont il apprécie davantage la perspective tout en critiquant les excès (un certain dogmatisme), ou encore de Renan auquel il voue une réelle admiration, et qui échappe davantage à ses critiques. Sans doute faut-il en chercher la cause dans le refus double de Renan qui récuse à la fois le matérialisme et les croyances religieuses : il tente de définir un idéalisme dégagé des hypothèses métaphysiques, et fondé sur la conscience que la seule loi du monde est un " éternel fieri, une métamorphose sans fin »: «tout se transforme " ${ }^{8}$. Dès 1863 il estime d'ailleurs que les hypothèses de Darwin sont «dans la voie de la grande explication du monde et de la vraie philosophie $»^{9}$. Flaubert partage avec lui ce sentiment qui l'éloigne du dogmatisme religieux comme scientifique: très tôt il manifeste le même sens de l'évolution, qu'elle soit naturelle ou historique, et si le progrès dont il se moque souvent aura sa place dans le Dictionnaire des idées reçues, ce n'est pas le cas de l'évolution. Tout change, même les savoirs et les représentations que l'on se fait du monde, ce qui conduit Flaubert à se méfier des conclusions, de la modalité assertive du discours. Il définit alors l'œuvre d'art comme un dispositif antidogmatique et sceptique : son seul objectif est de faire rêver le lecteur et de lui redonner le sens de l'infini ${ }^{10}$, un infini qui échappera toujours à la volonté de savoir ${ }^{11}$. Flaubert n'a pas attendu la lecture de Spencer - lui aussi fasciné par l'idée de l'infini - pour s'en faire une idée. Il avait lu assez tôt Spinoza qui avait dû lui en faire sentir l'impersonnalité et l'inaccessibilité ${ }^{12}$ dans l'Éthique, et Buffon qui avait pu lui en faire éprouver à la fois le vertige et la poésie dans Les Époques de la nature.

Dans les années 1870, même si Flaubert défend le positivisme en politique par réaction aux événements proches ${ }^{13}$, il dit par ailleurs souvent sa gêne d'un point de vue intellectuel à l'égard d'une pensée trop physiologiste ${ }^{14}$, et dogmatique. Les positivistes se sentaient obligés de se positionner fermement contre un spiritualisme qui ne voulait pas quitter la scène. Le débat durera, car si Victor Cousin meurt en 1867, la seconde génération de philosophes spiritualistes - Paul Janet ${ }^{15}$ et Marie-Elme Caro en particulier assure la relève et le spiritualisme reste dominant institutionnellement dans les années 1870-1880 ${ }^{16}$. Le positivisme est aussi par ailleurs vilipendé par les catholiques, en particulier par Monseigneur Dupanloup ${ }^{17}$. La réception de Spencer intervient dans un long débat qui a débuté dès la première moitié du siècle après la publication des travaux de Cabanis et Bichat tandis que Victor Cousin qui a conquis très vite une domination institutionnelle ${ }^{18}$ s'affronte à Broussais, élu comme lui et la même année (1832) à l'Académie des sciences morales et politiques. Ce débat prend toute son ampleur dans les années 1850 et encore davantage dans les années 1860 avec la traduction de l'Origine des 
espèces de Darwin (1862) et la création par Littré en 1867 d'un organe de presse dévolu au positivisme (La Philosophie positive).

7 La réception de Spencer intervient à un moment de forte tension entre les penseurs qui défendent la science et les spiritualistes ou les défenseurs de la religion. En 1863, Littré a tenté une élection à l'Académie française et Monseigneur Dupanloup a réagi en publiant le célèbre pamphlet Avertissement à la jeunesse et aux pères de famille sur les attaques dirigées contre la religion par quelques écrivains de nos jours qui vise Littré, Taine, Renan et Maury. En 1864, Taine riposte avec la préface et la thèse déterministe de l'Histoire de la littérature anglaise, tandis que Elme-Marie Caro publie L'idée de Dieu et ses nouveaux critiques (1864) qui connaîtra une dizaine de rééditions jusqu'à la fin du siècle. Le $1^{\mathrm{er}}$ juin 1864, Renan est révoqué du Collège de France à cause de son cours de 1862 et de la publication de la Vie de Jésus. Le premier article sur Spencer en France est publié dans cette période, en $1864^{19}$, par Auguste Laugel qui le situe par rapport à Auguste Comte dont il perçoit l'influence inavouée sur le philosophe anglais - alors que celui-ci s'en est toujours défendu ${ }^{20}$. Laugel cite le fait que Spencer refuse de trancher entre le matérialisme et le spiritualisme, et il souligne le paradoxe d'un curieux positivisme construit à la gloire de l'inconnaissable, qui donne des limites à la science, défend l'existence d'une inintelligibilité irréductible qui fait de l'Univers un problème insoluble: seuls les rapports entre les choses sont connaissables. L'article de Laugel suscite la même année la réaction critique de Littré contre Spencer dans son introduction (intitulée «Préface d'un disciple») à la nouvelle édition du Cours de philosophie positive (1864) de Comte, que Flaubert lira dès $1872^{21}$. Littré affirme que la «science, qui n'est devenue positive que depuis qu'elle expérimente et vérifie, ne veut plus d'une finalité qui ne se vérifie ni ne s'expérimente $»^{22}$. La science positive "ne poursuit ni les causes premières ni la fin des choses", elle "procède en établissant des faits et en les rattachant les uns aux autres par des relations immédiates ». Et il ajoute : «C'est la chaîne de ces relations, chaque jour étendue plus loin par les efforts de l'intelligence humaine qui constitue la science positive $»^{23}$. Littré attaque Spencer et sa pensée de «l'immensité inconnue $»^{24}$ en faisant remarquer que l'incogniscible qui n'est pas l'inconnu était une idée formulée par Comte, mais qu'elle avait chez lui le mérite d'échapper à la métaphysique. Selon Littré, Spencer mélange deux choses différentes: l'incogniscible de la science (lié à ses limites) et l'inconnu qui est l'objet d'une foi religieuse. Littré reconnaît que l'ampleur du temps et de l'histoire, que la solitude de l'homme face aux seules lois qui régissent l'univers lui donnent un sentiment d'immensité, mais il récuse la glorification spencérienne de l'inconnaissable. La réception de Spencer débute donc par un débat sur son rapport à Auguste Comte. En 1864, Littré n'a pas encore lu les Premiers principes mais il réagit sur un point du compte rendu de Laugel: la science et la religion - remarque Laugel - n'ont plus de raison pour Spencer de s'opposer en ennemies car elles buttent toutes les deux sur l'inconnaissable. Selon Littré, c'est sur ce point que Spencer est infidèle au positivisme d'Auguste Comte. Quoi qu'il en soit, comme Laugel, Littré reconnaît le grand intérêt de Spencer ${ }^{25}$, le désignant ainsi à l'attention des lecteurs. Émile Cazelles, traducteur en 1871 des Premiers principes fera précéder le texte de Spencer d'une longue présentation qui souligne l'intérêt de ce système et lui donne tout son relief dans les réflexions de l'époque.

Dans les années 1870, qui voient à la fois la multiplication des traductions de Spencer et des publications dans les deux camps opposés des spiritualistes et des positivistes, le débat se focalise sur la question du finalisme auquel les positivistes opposent l'idée d'évolution, également centrale dans la pensée de Spencer, dans celle de Renan et de 
Flaubert. La philosophie de Spencer suscite à la fois un intérêt et des critiques dans les deux camps, car elle semble proposer une troisième voie. Mais Spencer n'est pas le seul qui voudrait trouver une tangente tout en mettant au point une pensée totalisante qui ressaisisse de manière évolutionniste l'histoire humaine et l'histoire de la nature : Renan - qui correspond d'ailleurs avec Spencer ${ }^{26}$ - en avait fait à sa façon une tentative dès 1863 , qui avait suscité en retour une réponse strictement positiviste de Marcellin Berthelot ${ }^{27}$.

Dans les années 1860-1870, Flaubert fréquente des positivistes (Littré28, Taine, Baudry, Maury, Vacherot ${ }^{29}$, des scientifiques qu'il croise par exemple aux dîners Magny dans les années 1860 (Claude Bernard, Marcellin Berthelot ${ }^{30}$ ), et des penseurs plus difficiles à classer comme Renan ${ }^{31}$ qui ne se reconnaît pas lui-même comme un positiviste, mais qui fait néanmoins partie d'une nébuleuse de penseurs qui défendent la science et un esprit nouveau. Il connait aussi dans l'autre camp les travaux des spiritualistes libéraux, Paul Janet en particulier, et il a lu Victor Cousin et Elme-Marie Caro qu'il n'apprécie pas ${ }^{32}$. Bref, il lit les arguments des uns et des autres, et il est en contact direct avec des milieux qui débattent de questions impliquées dans les discussions sur Spencer à partir de 1864.

La préparation de Bouvard et Pécuchet concorde avec cette période de polémique et de réception de Spencer. Or, Flaubert met en scène dans son roman deux personnages férus de science, et convaincus que celle-ci peut atteindre des certitudes, trouver des lois scientifiques stables et définitives, l'origine de tous les phénomènes, des causes finales positives! L'interrogation sur les causes finales est récurrente dans les dossiers préparatoires de Bouvard et Pécuchet. Dès l'étape des lectures et des notes documentaires, Flaubert multiplie les rubriques sous ce titre ${ }^{33}$, et il constitue des folios de notes sur le livre de Paul Janet, qui sera toutefois absent de la bibliothèque des deux bonshommes, tout comme les travaux de Darwin et de Spencer, puisque l'intrigue est située à la fin des années 1840 et au début des années 1850. Mais, dans le roman, les deux personnages refusent d'admettre les limites de la science et s'obstinent à méconnaitre l'existence de ce que Spencer appelle "l'inconnaissable ». Le philosophe anglais défend l'idée d'une cause première (rejetée par contre par le positivisme de Littré) tout en insistant sur le fait qu'elle demeure hors de portée de l'esprit humain. Les Premiers principes s'ouvrent avec une grande partie sur l'inconnaissable. Celle-ci débute par un chapitre de confrontation entre science et religion et se clôt par un chapitre intitulé « Réconciliation ».

Lorsqu'on sait l'agacement de Flaubert à l'égard des dualismes, à l'égard du matérialisme, nouvelle religion à rebours, à l'égard des prétentions anthropocentriques et des certitudes, on n'est pas étonné de découvrir en 1878 cette charge contre une pensée inconséquente: "Les positivistes français se vantent: ils ne sont pas positivistes! Ils tournent au matérialisme bête, au d'Holbach! Quelle différence entre eux et un Herbert Spencer! Voilà un homme, celui-là ! De même qu'on était autrefois trop mathématicien, on va devenir trop physiologiste. $\aleph^{34}$ Dans cette lettre, Flaubert insiste sur la différence entre le véritable positivisme - un esprit positif qui remet inlassablement en cause ses hypothèses et affronte courageusement l'inconnaissable - et le positivisme triomphant de la seconde moitié du siècle. Le modèle mathématique (modèle d'évidence que l'on atteint par la spéculation) caractérisait le cartésianisme auquel le spiritualisme du xix ${ }^{e}$ siècle reprend des éléments, tandis que le positivisme de la seconde moitié du siècle verse dans un déterminisme matérialiste qui pour se différencier de celui des causes finales n'en est pas moins tenté par une forme de pensée conclusive qui rebute tout autant Flaubert. Il n'adhère à aucune des deux orientations dominantes de son époque: depuis la fin des années 1850, il conteste dans sa Correspondance l'opposition entre matérialisme et 
spiritualisme. Pour autant, on ne trouvera jamais de trace chez Flaubert d'une réconciliation de la religion et de la science. En 1849, La Tentation mettait en scène sur un mode critique la fascination de la Science pour la Foi, et dans Bouvard et Pécuchet la tentation de la conversion échoue, tandis que le roman tourne en dérision la posture religieuse des deux faux savants qui demandent des croyances à la science.

Après 1870, dans les années où les textes de Spencer sont traduits et où on en débat de plus en plus, deux nouveaux éléments interviennent. D’un côté, la défaite française est parfois attribuée à une infériorité par rapport à l'avancement de la science en Allemagne. En 1871, dans La Réforme intellectuelle et morale Renan fait de la science la base d'un renouvellement national et contre le suffrage universel, il affirme la supériorité du savant et du noble, idée que l'on retrouve dans le rêve flaubertien d'un gouvernement de mandarins ${ }^{35}$. Toutefois, malgré une valorisation de la science, on n'en est plus aux rêves du premier positivisme comtien, dont les positivistes de la nouvelle génération refusent eux-mêmes les dérives utopistes et optimistes. Les positivistes ont été rejoints par des scientifiques (Charles Robin ${ }^{36}$, Marcellin Berthelot, Claude Bernard) ce qui semble donner une légitimité à ce courant. D'un autre côté, dans le même temps, Renan évolue de plus en plus vers un scepticisme notable dans Les Dialogues philosophiques (1871) ou dans La Prière sur l'Acropole de 1876, admirée par Flaubert. C'est dans ces mêmes années 1870, que le débat sur Spencer s'amplifie. À partir de 1872, la revue fondée par Littré (et Grégoire Wyrouboff), La Philosophie positiviste, publie plusieurs réactions des positivistes qui attaquent Spencer principalement sur deux points: son évolutionnisme qu'ils considèrent comme une déviance métaphysique ${ }^{37}$ parce que la force à l'origine de l'évolution demeure une pure hypothèse; le naturalisme de sa philosophie met sur le même plan l'évolution sociologique et l'évolution biologique ${ }^{38}$, méconnaissant ainsi la particularité des faits humains. Dans l'autre camp, les défenseurs du spiritualisme l'attaquent sur un autre flanc, tout en se montrant intéressés par certaines de ses idées.

Le livre de Paul Janet - Les Causes finales (1876) - donne une idée des questionnements de l'époque. Il résume les positions diverses, il réfute les idées positivistes, il critique la conception de forces purement mécaniques, il attaque Darwin qui n'élimine d'ailleurs pas selon lui toute finalité et l'idée d'une direction plastique ; et il défend la thèse adverse de l'existence d'une cause intelligente à l'origine des choses et concevable par l'esprit, c'està-dire qu'il prend le contre-pied de Spencer. Paul Janet lui consacre un chapitre et résume les Premiers principes. Il insiste sur le refus spencérien de la notion de finalité au profit d'une conception de la conservation de la force. Il est question aussi de Spencer dans La Philosophie d'André Lefèvre (1879), qui est pour sa part plutôt du côté des matérialistes. Flaubert cite à plusieurs reprises ce livre dans le dossier documentaire de Bouvard et Pécuchet, en particulier sur la question de Dieu et des causes finales ainsi que sur la notion de force ${ }^{39}$.

Spencer formule des idées susceptibles de modifier les termes du débat entre les spiritualistes et les positivistes en renvoyant dos à dos les deux camps, en rendant visible aussi un paradigme évolutionniste à partir duquel il devient possible de différencier l'esprit positif d'un positivisme trop dogmatique : il fournit donc quelques arguments qui permettent de retourner l'évolutionnisme contre les dogmatismes de toutes tendances et même contre le dogmatisme positiviste. Je ne retiens que les éléments de la philosophie de Spencer les plus caractéristiques d'un paradigme évolutionniste qui se forme dès les années 1850 et dont on peut percevoir des éléments chez Flaubert comme chez d'autres 
penseurs ${ }^{40}$ dans les années qui précédèrent la publication des grands best-sellers de l'évolutionnisme (Darwin, Spencer, Haeckel).

1) Spencer refuse avec vigueur l'opposition du matérialisme et du spiritualisme et lui préfère une conception dynamique du monde : il met au premier plan la conservation et la transformation de la " force ", mot récurrent dès la publication en 1855 des Principes de psychologie. Souvent éclectique dans ses lectures et volontiers syncrétique dans ses jugements, Flaubert a tiré de Spinoza ${ }^{41}$, Cabanis et Maury ${ }^{42}$ plus probablement que d'une lecture précoce de Spencer des objections contre le dualisme chrétien et même une pensée moniste. Ainsi il écrit en 1859 : « [...] je ne sais (et personne ne sait) ce que veulent dire ces deux mots : âme et corps, où l'un finit, où l'autre commence. Nous sentons des forces et puis c'est tout. Le matérialisme et le spiritualisme pèsent encore trop sur la science de l'homme pour que l'on étudie impartialement tous ces phénomènes. L'anatomie du cœur humain n'est pas encore faite. [...] Ce sera l'unique gloire du XIx siècle que d'avoir commencé ces études. Le sens historique est tout nouveau dans ce monde. On va se mettre à étudier les idées comme des faits, et à disséquer les croyances comme des organismes. Il y a toute une école qui travaille dans l'ombre et qui fera quelque chose, j'en suis sûr. $»^{43}$

2) La force échappe à la connaissance: «l'exercice de la force est complètement inintelligible ", reconnaît Spencer ${ }^{44}$. Elle est - commente Émile Cazelles - "une cause persistante d'effets fugaces, qui s'y manifeste par le fait même du changement $\aleph^{45}$. Cette conception permet de se débarrasser à la fois de l'idée de finalité et de l'idée de progrès. La force se conserve par transformation, elle œuvre dans la complexification du réel, dans le sens de l'hétérogénéité, et cela à tous les niveaux. Mais, par contre, elle ne va pas forcément dans la direction d'un mieux moral ou esthétique. Spencer intègre de surcroît dans son système l'idée de dissolution ${ }^{46}$. Cette pensée évolutionniste, s'appuie sur un organicisme dont Judith Schlanger a montré la prégnance au XIX ${ }^{e}$ siècle ${ }^{47}$. Spencer utilise le modèle de l'embryon qui se développe et meurt, et il signale d'ailleurs lui-même l'importance de l'embryologie du biologiste Karl Ernest von Baer dans la structuration de sa pensée ${ }^{48}$. C'est cette idée qui lui permet de concevoir un évolutionnisme moins finaliste que celui de Darwin. C'est probablement une pensée d'une part organiciste elle aussi et d'autre part lucrécienne qui sous-tend aussi la conception que Flaubert se fait de l'histoire cyclique comme un mouvement perpétuel d'accroissement et de désagrégation des civilisations et des races, dont témoigne la lettre terrible du 5 octobre 1870 à sa nièce Caroline sur la succession et la fin des races, qui semble aussi intégrer l'idée d'une lutte pour la vie lorsque Flaubert évoque les races se dévorant les unes les autres pour survivre et triompher, avec le sentiment que la défaite a sonné le glas des latins auxquels il appartient.

3) Mais chez Spencer, c'est moins un cycle, qu'un rythme qui est mis en évidence dans la conception de l'évolution/dissolution. D'ailleurs, sans le rythme il faudrait imaginer un infini vide ne contenant qu'un seul corps en mouvement, explique Spencer. Le rythme est à la fois une logique et ce qui se substitue à l'essence (comme le mouvement chez Héraclite). Il est lié à une pensée de la différenciation qui postule l'existence de forces opposées : le « rythme se produit partout où il y a un conflit de forces qui ne se font pas équilibre $»^{49}$. Le rythme est encore central dans les Essais de morale, de science et d'esthétique. Essais sur le progrès, traduits en 1877. Auguste Burdeau, le traducteur, insiste sur l'alternance évolution/dissolution qui le constitue ${ }^{50}$. Mais Spencer n'est pas l'inventeur de la notion philosophique de rythme (et pas davantage de la notion de 
contraste qui sous-tend sa pensée du rythme). La pensée du rythme remonte à l'Antiquité ${ }^{51}$, aux présocratiques (Héraclite et Démocrite) : elle est liée philosophiquement au « tout s'écoule » d'Héraclite (panta rhei), penseur auquel Flaubert se réfère explicitement dans le manuscrit de Salammbô pour énoncer sa conception de la vie et de l'histoire: "Tout meurt pour l'accomplissement de l'unité et tout renaît en elle. Au sein de l'unité, il faut que la multiplicité éclate - toute chose naît de l'opposition. Il n'y aurait pas de génération sans la guerre (Héraclite): ce qui s'oppose tend à l'accord. L'harmonie résulte de l'opposition de l'unité à elle-même. Tout cela coule comme un fleuve $\aleph^{52}$. Significativement, c'est dans le contexte d'une telle réflexion qu'apparaissait alors aussi l'expression « force suprême infinie " $^{53}$.

18 Émile Cazelles souligne dans l'œuvre de Spencer une pensée de la labilité du monde : les «courants de force » - écrit-il - «ajoutent du mouvement à la matière ou leur en enlèvent à chaque instant. Vue de haut, l'évolution, avec son corrélatif la dissolution, représente un rhythme immense d'une durée que l'imagination humaine ne peut embrasser. Tout ce qu'elle peut faire, c'est de se créer une représentation symbolique de cette série d'ondes qui porte notre monde de l'état d'extrême diffusion, antérieur à la formation des nébuleuses, à l'état d'équilibre dans la plus extrême concentration et le ramène ensuite par une désagrégation graduelle à son état primitif. ${ }^{54}$ L'évocation poétique du monde rythmé comme par une série d'ondes sera une objection de Bouvard à un moment où les deux personnages en quête du savoir, de la cause finale, se trouvent impuissants : «[...] des matières organiques ont pris part à la formation du silex, de la craie, de l'or peut-être ! Le diamant n'a-t-il pas été du charbon : la houille un assemblage de végétaux : - en la chauffant à je ne sais plus combien de degrés, on obtient de la sciure de bois, tellement que tout passe, tout coule. La création est faite d'une matière ondoyante et fugace. Mieux vaudrait nous occuper d'autre chose $! »^{55}$ Ils ont une réaction qui ressemble au vertige évoqué par Elme-Marie Caro dans son ouvrage sur les «nouveaux critiques de l'idée de Dieu». Pour eux, explique Caro, la réalité n'est plus qu'un "phénomène qui passe »; ils imaginent le «mouvement de la nature détruisant toute réalité fixe ; l'objet de la nature entrainé dans le torrent des phénomènes avec la pensée elle-même. $\|^{56}$ Après sa réflexion découragée, Bouvard s'endort, mais les deux personnages - qui ne sont vraiment pas des disciples de Spencer ni d'aucun autre évolutionniste - ne s'en tiendront pas là et repartiront de plus belle dans la recherche des causes finales.

19 La pensée du rythme est antidialectique et permet de faire l'économie du progrès - idée qui exaspérait Flaubert. Dans l'Introduction aux Premiers principes, Émile Cazelles met en valeur ce point important de la pensée de Spencer en lui confrontant la position de Renan dans la célèbre lettre ouverte adressée en 1863 à son ami Marcellin Berthelot : « [...] nous n'avons aucune raison de croire que dans le Tout le mouvement progressif prédomine sur le mouvement régressif, et que le progrès soit la loi de l'univers dans sa totalité $\aleph^{57}$. De fait, Renan qui est parfois sceptique - et qui le sera de plus en plus dans les années 1870 s'était laissé aller en 1863 à une rêverie sur le progrès et l'homme, parcelle de Dieu en devenir dans l'histoire de l'humanité. Or, le système de Spencer s'oppose à une telle conception. Dans un article de 1873 intitulé «Les métamorphoses de l'idée de progrès dans la science contemporaine $\aleph^{58}$, Elme-Marie Caro reconnaît cet avantage à la philosophie de Spencer : "il a contribué à nous délivrer de cette idolâtrie d'un progrès rectiligne, continu, illimité, dont l'apothéose insensée a égaré tant d'esprits depuis un siècle $~_{59}$. De son côté, dès 1857 , Flaubert avait fait cette réflexion : «L'infini, submerge 
toutes nos conceptions et, du moment qu'il est, pourquoi y aurait-il un but à une chose aussi relative que nous ? ${{ }^{60}}^{0} \mathrm{Ou}$ encore : "l'humanité [...] est toujours en marche et [...] elle ne conclut pas. / [...] je crois à l'évolution perpétuelle de l'humanité et à ses formes incessantes [...]. La démocratie n'est pas plus son dernier mot que l'esclavage ne l'a été, que la féodalité ne l'a été, que la monarchie ne l'a été. L'horizon perçu par les yeux humains n'est jamais le rivage, parce qu'au-delà de cet horizon, il y en a un autre, et toujours! $\aleph^{61}$ Spencer n'avait pas encore écrit ses Premiers principes ${ }^{62} !$ Mais l'historiographie du XIx ${ }^{\mathrm{e}}$ siècle (à propos de laquelle Flaubert était bien informé) avait déjà largement ouvert la perspective en brassant les siècles et les civilisations dans une longue durée, tandis que dans le domaine de l'histoire naturelle les premiers éléments transformistes avaient été ébauchés par Buffon dès la seconde moitié du XVIII ${ }^{\mathrm{e}}$ siècle $^{63}$, suivis par le transformisme de la Philosophie zoologique (1809): Lamarck y théorise l'évolution par complexification et diversification, ce qui sera encore une idée importante dans les Premiers principes de Spencer, même s'il préfère citer les travaux d'un biologiste plus moderne. Ajoutons que dans l'entourage de Flaubert, son ami Louis Bouilhet avait écrit en 1854 un grand poème évolutionniste avant la lettre (Les Fossiles), qui imaginait même la fin de l'homme supplanté par une espèce mieux adaptée au mouvement et à la puissance de la vie! Lecteur de Buffon et de Lamarck, qui ont employé la notion de force soit en un sens newtonien (Buffon), soit en un sens plus vitaliste (Buffon et Lamarck) afin de penser la nature sans miracles, Flaubert avait d'autant moins besoin de Spencer pour penser la loi suprême de l'évolution que, plus près de lui encore, son Louis Bouilhet l'avait lui-même imaginée dans Les Fossiles comme une « force cachée aux flancs de la nature » ${ }^{64}$, infinie et insensible à la destinée humaine.

Dans les années 1850 des idées circulent et constituent d'abord une nébuleuse instable. Une « école " travaille "dans l'ombre » ${ }^{65}$, dit Flaubert qui est attentif à ce mouvement. Dans cette période il fait aussi des lectures dans le domaine des sciences naturelles (Buffon, Cuvier, Geoffroy Saint-Hilaire), et a peut-être conseillé Louis Bouilhet dans la rédaction de son poème, selon leurs habitudes de collaboration. Il est par ailleurs l'ami de la famille Pouchet, rouennaise comme la sienne. Félix, le père, défend encore la génération spontanée qui reste attachée aux premières pensées transformistes (Lamarck l'admettait aussi), et il publie en 1859 Hétérogénie, un livre qui s'efforce de penser l'histoire naturelle en faisant l'économie du hasard et des interventions de la transcendance. Georges, le fils, naturaliste également, est le conseiller de Flaubert en matière de sciences naturelles. Lecteur de Lamarck, il écrit dans De la Pluralité des races humaines (1858): "Lamarck sentait bien qu'un temps infini est la condition de la variabilité illimitée ${ }^{66}$. Lecteur également de Gobineau, qu'il résume dans son livre, il pense que si la nature marche généralement de l'infériorité vers la perfection, il faut aussi admettre la possibilité d'une "évolution rétrograde ${ }^{67}$, idée qui caractérisera aussi l'évolutionnisme spencérien. Lorsque, dans les années 1850, Flaubert parle d'évolution perpétuelle, et rêve sur l'infini qui met en échec la raison humaine, il n'a donc besoin ni de Darwin ni de Spencer.

21 4) Spencer pense parfois en termes kantiens ${ }^{68}$ la relativité de la connaissance, l'évolutionnisme rejoignant alors un criticisme que Flaubert connait bien pour l'avoir fait pratiquer au Diable de La Tentation de saint Antoine en 184969). Dans les Premiers principes, Spencer écrit que l'explicable - qui est l'objet de la science - ne concerne que la genèse des choses « telles qu'elles se manifestent à la conscience humaine » mais qu'il y a au-delà de l'inintelligibilité au nom de laquelle il renvoie dos à dos lui aussi les Matérialistes et les 
Spiritualistes $^{70}$. Dès les années 1850 , le monde en lui-même est déjà insaisissable, et toujours perçu au travers des manières de voir. L'objectif et le subjectif ne se distinguent donc pas - ce qui est l'une des idées de Spencer - et aucune conception du monde n'est mieux fondée qu'une autre. Madame Bovary faisait dialoguer en vain le scientisme d'Homais et la foi de Bournisien. En 1868, Flaubert énonce un criticisme philosophique qui est aussi l'une des raisons pour lesquelles il renvoie dos à dos le matérialisme et le spiritualisme : «Je ne sais pas ce que veulent dire ces deux substantifs Matière et Esprit ; on ne connaît pas plus l'une que l'autre. Ce ne sont peut-être que deux abstractions de notre intelligence? Bref, je trouve le Matérialisme et le Spiritualisme deux impertinences égales. $\|^{71}$

Malgré le face-à-face qui se poursuit, dans les années 1860-1900 le paradigme évolutionniste remporte l'adhésion de nombreux penseurs, écrivains et scientifiques. Il devient séduisant par la puissance poétique qu'il recèle peut-être davantage que par la pensée systématique et globalisante dont le système de Spencer témoigne avec sa «loi mécanique du progrès universel » (par différenciation, passage du simple au complexe, de l'homogène à l'hétérogène) qui s'applique à l'univers, à la société, à l'individu, aux produits de la pensée, au langage. L'évolutionnisme dans sa version plus largement culturelle (au-delà des philosophies particulières), bricoleuse et souple qui emprunte quelques images, qui oublie certaines idées ou certaines articulations, et qui puise éventuellement à des sources anciennes remises au goût du jour dans de nouvelles configurations discursives, est à l'origine d'un renouvellement du merveilleux: un merveilleux scientifique susceptible de réenchanter différemment le monde. Michelet dans La Mer, Quinet dans La Création (1870), Zola dans Le Docteur Pascal, Haeckel - auteur plus tard encore des Merveilles de la vie -, écrivains, historiens, scientifiques, tous cèdent à la fascination devant la force du monde et ses mystères. Avant Spencer, Michelet parle d'une « force de vie ${ }^{72}$ en 1861 . Se souvient-il d'un vitalisme dont l'évolutionnisme diffus des années 1860 reprendrait quelques éléments malgré le soupçon métaphysique qui pesait sur l'idée de force vitale défendue au XviII siècle? Quoi qu'il en soit, il ne semble alors pas absolument utile de chercher une relation intertextuelle précise avec l'œuvre de Spencer lorsqu'on voit apparaître le terme de force dans Bouvard et Pécuchet, après l'avoir vu dans la Correspondance de 1859, et dans l'avant-texte de Salammbô:

Une lisière de mousse bordait un chemin creux, ombragé par des frênes dont les cimes légères tremblaient. Des angéliques, des menthes, des lavandes exhalaient des senteurs chaudes, épicées; l'atmosphère était lourde ; et Pécuchet, dans une sorte d'abrutissement, rêvait aux existences innombrables éparses autour de lui, aux insectes qui bourdonnaient, aux sources cachées sous le gazon, à la sève des plantes, aux oiseaux dans leurs nids, au vent, aux nuages, à toute la Nature, sans chercher à découvrir ses mystères, séduit par sa force, perdu dans sa grandeur ${ }^{73}$.

Pécuchet touche pour une fois à ce qui fonde l'entreprise flaubertienne et que le roman met en abyme : l'inconnaissable. Mais les personnages voudraient le réduire tandis que la poétique du roman le fait inlassablement ressurgir. Les deux faux savants s'attaquent à des disciplines qu'ils ne connaissent pas. Or, ils font l'expérience - décrite aussi par Spencer - d'une réalité complexe qui ne livrera jamais son dernier mot parce qu'elle nécessite l'approche multiple de sciences connexes. Il n'est pas de science ultime qui puisse donner accès à la cause finale. Contre Comte dont il critique l'ordre « sériaire $»^{74}$, Spencer pense le classement des sciences comme un buissonnement dont la complexité est à la mesure de celle du monde $\mathrm{e}^{75}$. La connexité des sciences qui s'articulent les unes aux autres motive souvent le passage d'une discipline à l'autre dans certains chapitres de 
Bouvard et Pécuchet. La logique narrative de ce roman bien peu romanesque rend compte d'une complexité inépuisable du réel, tant par l'enchaînement des disciplines que par la structure répétitive qui fait alterner recherches et échecs sans solution dialectique dans une temporalité entropique qui loin de construire une histoire de la connaissance et de ses progrès se contente de faire diminuer les ressources budgétaires des deux personnages et leur crédit auprès des Chavignollais. Le savant efficace selon Spencer est celui qui est conscient des limites de sa discipline, et qui, plus largement, est même conscient de ce qui déborde irréductiblement notre esprit ${ }^{76}$. Bouvard et Pécuchet ne sont ni conscients de leurs propres limites ni de celles des sciences qu'ils investissent d'un désir dogmatique, et quasiment religieux, en leur demandant une croyance digne de foi plus que des connaissances relatives et utiles.

Flaubert construit la poétique de ce roman contre le progrès, les causes finales, l'idée de but - qui sont au centre des interrogations des personnages. Il crée un récit critique à l'égard de la notion de causalité et qui progresse souvent lui-même par des hasards ou des glissements entre sciences connexes. Pour donner une consistance et une construction à ce type de récit, Flaubert réfléchit dans ses scénarios sur un nouveau mode de logique, purement textuel : une logique rythmique. Sur un folio intitulé «Notes» sous le titre "Méthode", il écrit: «À partir du chapitre III, Sciences, rattacher au personnage secondaire qui paraît dans chaque chapitre les personnages tertiaires, [...] et le nombre des personnages tertiaires doit aller en augmentant à mesure qu'on approche de la fin. $\gg^{77}$ Deux rythmes structurent le roman: d'une part la répétition (quête/échec, illusion/ désillusion, construction/déconstruction), et d'autre part une sorte d'accélération et d'emballement sur la fin du roman, mais ce mouvement sans but, sans espoir de connaissance tourne à vide, il est purement rythmique.

La dynamique répétitive et rythmique du roman convertit en poétique romanesque une nébuleuse d'idées qui ont traversé la Correspondance d'année en année et dans lesquelles Flaubert reconnaît après coup le paradigme évolutionniste lorsque sa lecture de Spencer lui en offre la réalisation philosophique. Aussi peut-on comprendre son enthousiasme «Voilà un homme celui-là !» - et en même temps son silence. Était-il nécessaire de commenter et de prendre des notes sur ce qu'il avait déjà très bien pensé par lui-même, mais à bâtons rompus et en lisant une multiplicité de textes, en bricolant diverses idées qui vont dans le sens d'un scepticisme et d'une conception définalisée de l'« évolution perpétuelle » qu'il a élaborés sans Spencer, dès les années 1850. Notons tout de même au profit de Spencer que Flaubert, généralement rebuté par les systèmes - auxquels il préfère la synthèse, une " harmonie des choses disparates $»^{78}-$, semble désarmé dans ses réticences par le système du philosophe anglais lorsqu'il le place au-dessus des positivistes français. Probablement parce que ce système était paradoxalement fondé sur l'inconnaissable et sur une conception des «limites de la science ». Sans doute Flaubert pouvait-il admirer ce tour de force d'un système construit contre les systèmes, d'un dogmatisme antidogmatique.

Flaubert s'enthousiasme en 1878 pour Spencer, parce que sa propre pensée s'est infléchie sans le savoir dans cette direction au contact des débats de son époque. L'évolutionnisme de Spencer fait signe vers un modèle de pensée et une vision du monde qui correspondent à un paradigme différent par rapport au physiologisme matérialiste dont Flaubert 
regrette la prédominance. Spencer a structuré en système philosophique un certain nombre d'idées qui ne trouvaient d'ancrage ni du côté spiritualiste ni du côté positiviste, des idées qui, considérées séparément, n'étaient pas nouvelles, ce qui faisait dire au biologiste et philosophe Thomas Huxley: "C'est le plus original des penseurs, bien qu'il n'ait jamais inventé une pensée nouvelle. Il ne lit jamais, il amasse simplement ce qui l'aidera à illustrer ses théories. $\gg^{79}$ Penseur original sans pensée nouvelle, Spencer puise les éléments de sa philosophie dans une nébuleuse idéologico-épistémologique qui intègre des idées libérales sur la primauté de l'individu, la sélection du plus apte, les limites de la science, l'inconnaissable, l'évolution en lieu et place du progrès, des idées dont il n'est pas l'inventeur mais qu'il articule en un système nouveau et qui a une force de globalisation.

La présence en pointillé du paradigme évolutionniste dans la Correspondance de Flaubert à partir du milieu des années 1850 est concevable, sans qu'il soit indispensable de la valider à partir d'une intertextualité spencérienne. Les années 1850 sont des années en quelque sorte pré-paradigmatiques. Thomas Kuhn conçoit l'histoire des paradigmes comme une lutte sur un modèle darwinien: un paradigme cède la place à un autre lorsqu'il ne répond plus efficacement aux objections, qui au lieu de stimuler de nouvelles recherches de l'intérieur demeurent alors tout à fait extérieures au paradigme. Cette conception qui permet de comprendre des changements dans un domaine disciplinaire ne prend toutefois pas en compte l'interaction des domaines, la circulation des idées et leur hybridation dans un champ plus large, moins délimité par l'appartenance à une communauté scientifique. Or, au XIX ${ }^{e}$ siècle lorsque philosophie et science dialoguaient et croisaient ensemble d'autres domaines des sciences humaines ainsi que la littérature, la formation d'un paradigme comme le paradigme évolutionniste ne pouvait s'expliquer dans le contexte du seul domaine de la biologie et de ses apories scientifiques, à partir de débats dans le champ clos d'un savoir spécialisé. La conception à la fois d'une période préparadigmatique et d'une généralisation du paradigme à la croisée des disciplines permet d'envisager de façon plus complexe la genèse des paradigmes, de manière plus interdisciplinaire également, ce qui est important dans le cas d'un paradigme qui a franchi - on le sait - les frontières disciplinaires. Une telle conception de la formation des paradigmes peut aussi dans le cas de la littérature, et plus particulièrement de Flaubert, expliquer la présence d'une nébuleuse d'idées qui ne sont d'ailleurs pas toutes contemporaines, qui sont liées à des lectures diverses et même hétéroclites, à la fréquentation aussi de réseaux.

\section{NOTES}

1. Le dossier de Bouvard et Pécuchet cite seulement De l'éducation intellectuelle, morale et physique que Flaubert préfère à celui du docteur Charles Robin, un ami positiviste, qui avait publié avec Littré un Dictionnaire de médecine en 1863, et qui codirigeait la société de biologie avec Claude Bernard. Le " paquet de Spencer » qu'il réclame à Maupassant dans une lettre de février 1880 laisse supposer qu'il ne s'en est pas tenu à l'ouvrage sur l'éducation. Peut-être a-t-il lu Les 
Premiers principes ou Les Essais de science, de morale et d'esthétique dont le titre pouvait susciter sa curiosité et qui contenait un essai sur le progrès.

2. Dans son article, publié dans ce même numéro, Yvan Leclerc indique quelques raisons (ses relations avec Juliet Herbert, l'institutrice de sa nièce Caroline, et sa connaissance de l'anglais) mais reconnaît aussi qu'aucune preuve ne peut être apportée.

3. Lettre à Edma Roger des Genettes du 12 janvier 1878, Correspondance, édition établie par Jean Bruneau et Yvan Leclerc pour le volume V, Paris, Gallimard, coll. "Bibliothèque de la Pléiade ", 2007, V, p. 347.

4. La Correspondance et les Carnets de travail de Flaubert ainsi que les dossiers documentaires de Flaubert ne seront publiés qu'après sa mort, et les œuvres publiées ne disent rien des réflexions personnelles de l'auteur, impersonnalité oblige.

5. Voir « Un rôle pour l'histoire », La Structure des révolutions scientifiques, Paris, Flammarion, 1972, p. 17-27.

6. «La conclusion, la plupart du temps, me semble acte de bêtise. C'est là ce qu'ont de beau les sciences naturelles: elles ne veulent rien prouver. Aussi quelle largeur de faits et quelle immensité pour la pensée! Il faut traiter les hommes comme des mastodontes et des crocodiles. » (lettre à Louise Colet du 31 mars 1853; Correspondance, édition de Jean-Bruneau, Paris, Bibliothèque de la Pléiade, II, p. 294).

7. Lettre à Louise Colet du 2 juillet 1853, ibid., p. 370.

8. Renan l'écrira en 1883 dans ses Souvenirs d'enfance et de jeunesse (Euvres complètes, édition établie par Henriette Psichari, Paris, Calmann-Lévy, 1948, II, p. 846), mais cette idée oriente son travail d'historien des religions, son approche à la fois de la nature et des faits humains comme on le voit dans sa lettre de 1863 à Marcellin Berthelot : «Les sciences de la nature et les sciences historiques » (publiée dans la Revue des Deux Mondes, le 15 octobre).

9. Ibid., Cuvres complètes de Renan, édition établie par Henriette Psichari, Paris, Calmann-Lévy, 1948, I, p. 638.

10. Il le dit à Louise Colet dans sa lettre du 26 août 1853 ; Correspondance, II, op. cit., p. 417.

11. De ce point de vue, l'entreprise romanesque de Flaubert se distinguera radicalement de celle de Balzac, alors même que dans les années 1850 il partage la même admiration pour Geoffroy Saint-Hilaire et Cuvier (lettre à Louise Colet du 7-8 juillet 1853), cités par Balzac dans l'Avantpropos de La Comédie humaine comme les modèles de l'historien des mœurs, fondateur de l'étude des mœurs et « instituteur des hommes».

12. Spinoza développe l'idée d'une substance une et infinie, inaccessible en tant que telle à l'esprit en dehors de ses attributs et de ses modes.

13. C'est le cas de cette lettre qu'il envoie à George Sand après la fin de la Commune de Paris et l'instauration de la Troisième République : «Je crois, comme vous, que la République bourgeoise peut s'établir. Son manque d'élévation est peut-être une garantie de solidité ? C'est la première fois que nous vivons sous un gouvernement qui n'a pas de principe. L'ère du Positivisme en politique va commencer?» (25 juillet 1871, Correspondance, IV, op. cit., p. 352).

14. Dans le positivisme français de la seconde moitié du siècle, il faut signaler le poids de l'héritage des travaux de la première moitié du siècle aussi bien philosophiques que scientifiques, de Cabanis, Bichat et Broussais (qui s'oppose à Cousin dans les années 1830-1840).

15. Paul Janet s'affronte directement au positivisme en publiant par exemple La Crise philosophique. MM. Taine, Renan, Littré, Vacherot, Paris, Germer Baillière, 1865.

16. C'est Paul Janet qui occupe la chaire de philosophie à la Sorbonne.

17. Voir Léo Freuler, La crise de la philosophie au XIXe siècle, Paris, Vrin, 1997, p. 73-74.

18. Professeur à la Sorbonne où il donne en 1818 son célèbre cours Des idées $d u$ vrai, $d u$ bien et du beau, il est même un moment ministre de l'Instruction publique sous la Monarchie de Juillet, puis à partir de 1840 président de l'agrégation de philosophie, poste clef pour contrôler l'orientation de la philosophie, du moins dans l'enseignement. 
19. Revue des Deux Mondes, janvier-février 1864, p. 930-957.

20. En juillet 1854, après la lecture des textes de Comte, il publie dans la revue British Quarterly le premier article d'une série de textes visant à le différencier de Comte. Cet essai sur la genèse des sciences sera ensuite repris dans La Classification des sciences, et traduit en français dans la Revue scientifique en 1872 sous le titre «Pourquoi je me sépare de la philosophie d'Auguste Comte ».

21. Voir le $\mathrm{f}^{\circ} 64 \mathrm{v}^{\circ}$ du Carnet 15 qui donne une liste de livres lus entre août et octobre 1872, Carnets de travail, édition établie par Pierre-Marc de Biasi, Paris, Balland, 1988, p. 510.

22. Principes de philosophie positive par Auguste Comte, précédé de la Préface d'un disciple par É. Littré, $2^{\mathrm{e}}$ édition, Paris, J.-B. Baillière et fils, 1868, p. 35.

23. Ibid., p. 49.

24. Ibid., p. 57.

25. Ibid., p. 58-65.

26. Il serait donc peut-être aussi intéressant dans l'autre sens d'envisager du point de vue de Spencer l'impact de la pensée de Renan. Sur l'échange de lettres entre Spencer et Renan voir Patrick Tort, Spencer et l'évolutionnisme philosophique, Paris, PUF, 1996, coll. « Que sais-je? ».

27. À la lettre publique que lui a adressée Renan sous le titre «Les sciences de la nature et les sciences historiques ", le chimiste Marcellin Berthelot répond aussitôt dans la Revue des Deux Mondes par une lettre intitulée «La science idéale et la science positive » (texte ensuite repris par Berthelot dans Science et philosophie en 1883, et donné par Henriette Psichari à la suite du texte de Renan dans le tome I des CEuvres complètes, op. cit., p. 633-671).

28. Il voit Littré aux dîners Magny, il dîne aussi parfois avec lui et d'autres amis, en mars 1868, par exemple, il est invité avec lui, Renan et Maury chez Alfred Baudry (lettre à Jules Duplan, 14 mars 1868, III, p. 735).

29. Sur les réseaux positivistes voir Daniel Becquemont et Laurent Mucchielli, Paris, PUF, 1998, Le Cas Spencer. Religion, science et politique, Paris, PUF, 1998, p. 230-236, coll. « La Philosophie hors de soi ».

30. Voir Robert Baldick, Les dîners Magny, Paris, Denoël, 1972.

31. En 1863, Monseigneur Dupanloup le place parmi les positivistes auxquels s'attaque son Avertissement à la jeunesse et aux pères de famille sur les attaques dirigées contre la religion par quelques écrivains de nos jours. Cet ancien du séminaire Saint-Nicolas du Chardonnet (où enseignait Dupanloup qui fut son professeur) publie d'ailleurs la même année La Vie de Jésus qui fait scandale auprès des catholiques, Jésus n'étant plus considéré comme le fils de Dieu, mais un grand homme, maître de morale, comparable à de grands philosophes de l'antiquité. L'évêque d'Orléans réagit en organisant une expiation publique! Sur Renan voir l'article d'Annie Petit, «Le prétendu positivisme de Renan ", La Réception du positivisme, textes réunis par Michel Bourdeau, Revue d'Histoire des Sciences Humaines, Lille, Presses du Septentrion, 2003, p. 73-102.

32. Lettre à George Sand du 29 avril 1870 (Correspondance, IV, op.cit., p. 184) et lettre à Mme Tennant du 16 décembre 1879 (Correspondance, V, op. cit., p. 768).

33. Voir le site Les dossiers de Bouvard et Pécuchet de l'École Normale Supérieure de Lyon : http:// www.dossiers-flaubert.fr/ Un moteur de recherche permet de retrouver tous les folios consacrés à ce sujet.

34. Lettre à Edma Roger des Genettes du 12 janvier 1878 ; Correspondance, V, p. 347.

35. Lettre à George Sand du 30 avril 1871 ; Correspondance, V, op. cit., p. 314.

36. Médecin, physiologiste cofondateur en 1848 de la Société de biologie qu'il dirige avec Claude Bernard, il publie en 1863 avec Littré un Dictionnaire de médecine.

37. Voir par exemple l'article de J. D. Bell, « Religion et Science. Examen d'Herbert Spencer », La Philosophie positive, juillet-décembre 1870, t. VII, p. 345-380.

38. P. Pichard, «Étude de la sociologie par Herbert Spencer », La Philosophie positive, 1875, t. XIV, p. 14. 
39. Manuscrit conservé à la Bibliothèque municipale de Rouen, g 226, vol. 6, $\mathrm{f}^{\circ} 63 \mathrm{r}^{\circ}$. Dossiers de Bouvard et Pécuchet, op. cit.

40. En 1863, dans sa lettre ouverte à Marcellin Berthelot («Les sciences de la nature et les sciences historiques »), Renan signale après coup la présence d'une perspective évolutionniste dans ses travaux dès la fin des années 1840 , et il va même jusqu'à imaginer qu'il aurait pu aboutir aux mêmes conclusions que Darwin s'il s'était lui-même engagé dans la voie des sciences de la nature qui l'avaient tant attiré pendant ses années de formation.

41. Spinoza s'écarte du dualisme cartésien et chrétien de l'âme et du corps, et il permet de penser l'unité de l'homme : le corps et l'esprit renvoient à une même chose perçue sous deux attributs différents (étendue et pensée).

42. Cabanis qui est l'auteur des Rapports du physique et du moral (1802) formule plutôt un monisme matérialiste qui rattache les phénomènes de la pensée à l'influence du corps, tandis que les travaux d'Alfred Maury insistent aussi sur la somatisation et l'impact des idées, des désirs sur le corps et les troubles physiques.

43. Lettre à Mlle Leroyer de Chantepie du 18 février 1859 ; Correspondance, III, op. cit., p. 17.

44. Les Premiers principes, trad. Émile Cazelles, Paris, Germer Baillière, 1971, p. 63.

45. Ibid., résumé de Cazelles, p. XXVIII.

46. «Toutes les choses grandissent ou dépérissent, accumulent de la matière ou l'usent, s'intègrent ou se désintègrent. » (Ibid., p. 302).

47. Les Métaphores de l'organisme, Paris, Vrin, 1971.

48. Il lui emprunte aussi l'idée d'une évolution de l'homogénéité indéfinie à une hétérogénéité cohérente et précise ce qui lui permet de penser l'évolution dans le sens d'une complexification : «Reprenant une idée émise par Harvey, Volff et Baer ont démontré que pendant son évolution tout organisme passe d'un état d'homogénéité à un état d'hétérogénéité » (Les Premiers principes, op.cit., p. 360) et il insiste sur le rôle structurant qu'a eu cette idée sur son propre système : «C'est en 1812 que j'ai connu la manière dont Baer exprimait ce principe général. L'universalité de la loi a toujours été pour moi un postulat emportant avec lui la croyance correspondante, tacite sinon avouée à l'unité de procédé dans toute la nature. La proposition que toute plante et tout animal, originellement homogènes, deviennent graduellement hétérogènes, établit une coordination dans une foule d'idées non organisées, ou imparfaitement organisées. » (p. 360).

49. Les Premiers principes, op. cit., p. 273.

50. Essais de morale, de science et d'esthétique, I. Essais sur le progrès, trad. Par M. A. Burdeau, Paris, Germer Baillière, 1877, p. XII.

51. Voir de Pierre Sauvanet, Le rythme grec d'Héraclite à Aristote, Paris, PUF, 1999.

52. Voir N.a.fr. 23660 (manuscrit conservé à la BnF), fo 98. Cette «leçon» philosophique disparaîtra bien sûr pour se réinvestir dans la structuration du roman.

53. «La mort et la vie, la destruction et l'enfantement, la douleur et la joie, tout s'égalisait, tout s'absorbait, dans le feu, dans une force suprême infinie, inaccessible à la pitié et indifférente à la vertu » (N.a.fr. 23658, fo $163 \mathrm{v}^{\circ}$ ).

54. Les Premiers principes, op. cit., p. LX.

55. Bouvard et Pécuchet, édition établie par Stéphanie Dord-Crouslé, Paris, Flammarion, 1999, p. 147 , coll. « GF ».

56. L'idée de Dieu et ses nouveaux critiques, Paris, Hachette, 1864, p. 29.

57. Les Premiers principes, op. cit., p. LXI.

58. Revue des Deux Mondes, sept.-oct. 1873, t. 107, p. 743-774.

59. Revue des Deux Mondes, 1873, n 107, p. 774.

60. Ibid.

61. Lettre à Mlle Leroyer de Chantepie du 18 mai 1857 ; Correspondance, II, op. cit., p. 719.

62. Mais il a publié en 1857 , un essai sur le progrès. 
63. Voir en particulier Les Époques de la nature (1778): Buffon pense l'influence du milieu en particulier sur les évolutions de l'espèce humaine. Il pense l'œuvre du temps et la formation de l'univers, du monde et de la nature dans une longue durée, grâce à un travail lent qui s'oppose à l'idée créationniste. Il n'est pas étonnant que Buffon se soit heurté à Linné (défenseur de la fixité des espèces) et que la Sorbonne ait exigé une rétractation après la publication des Époques de la nature.

64. Les Fossiles, repris dans Poésies, Milon-la-Chapelle, Éditions H \& D, 2009, p. 203.

65. Lettre à Mlle Leroyer du 18 février 1859, op. cit.

66. De la pluralité des races humaines, Paris, J. B. Baillière, 1864, p. 173. Il s'appuie sur un passage du Système des animaux sans vertèbres, Paris, Deterville, 1801, p. 13.

67. Ibid., p. 187.

68. Émile Cazelles le souligne d'ailleurs dans son introduction lorsqu'il commente le « principe de relativité de la connaissance » : « on fait profession de ne rien savoir de positif sur la nature des changements survenus dans le noumène, et [...] on reconnaît la radicale impuissance de l'esprit à pénétrer le mystère de "l'existence inconditionnée" qui demeure dans notre conscience comme un corps auquel nous ne pouvons donner, quand nous voulons nous le représenter, que des formes qui soient nôtres, sans pouvoir jamais déterminer celles qui lui appartiennent réellement.» (op.cit., p.XXVII). Marie-Elme Caro taxe aussi de kantisme le criticisme des positivistes qu'il attaque dans L'Idée de Dieu et ses nouveaux critiques, op. cit.

69. Voir G. Séginger, «La Tentation et les savoirs ", revue Flaubert, revue critique et génétique, mis en ligne le 19 janvier 2009, http://flaubert.revues.org/389 (consulté le 22 mai 2014).

70. Les Premiers principes, op. cit., p. 79.

71. Lettre à sa nièce Caroline du 23 mars 1868 ; Correspondance, III, op. cit., p. 738.

72. La Mer, édition établie par Jean Borie, Paris, Gallimard, 1983, p. 76, coll. « Folio ».

73. Bouvard et Pécuchet, op. cit., p. 147.

74. Spencer, Classification des sciences [1869], Alcan, 1923, p. 93. Spencer refuse à la fois la succession des trois états (théologique, métaphysique, positif) et la classification des sciences elle aussi fondée sur une historicité, leur accession à la positivité se faisant en fonction de leur degré de complexité et de leur proximité par rapport à l'homme (la physique avant la chimie, avant la biologie).

75. Daniel Becquemont oppose à la classification comtienne, fondée sur une successivité, l'« arbre buissonnant et en expansion » de la classification complexe de Spencer avec des sciences qui se subdivisent et s'articulent entre elles (" Positivisme et utilitarisme : Comte, Spencer, Huxley », La Réception du positivisme, op.cit., p. 63). Malgré la distinction entre sciences abstraites, sciences abstraites concrètes et sciences concrètes l'interaction continue entre ces trois classes de sciences donne cette complexité dont Becquemont rend compte par la métaphore du buissonnement. Il cite pour éclairer la particularité cet extrait : «Depuis le commencement les sciences abstraites, les sciences abstraites concrètes, et les sciences concrètes, ont progressé ensemble, les premières résolvant des problèmes que posaient les secondes et les troisièmes, et se développant par la solution des problèmes; et les secondes se développant de la même manière en se joignant aux premières pour résoudre les problèmes posés par les troisièmes. Il y a toujours une interaction continue entre les trois grandes classes de sciences [...]. » (Essays, 1874, III, p. 71, cité par Daniel Becquemont, op. cit., p. 63).

76. La science nous fait toucher des bornes que nous ne pouvons franchir, explique Spencer dans De l'éducation intellectuelle, morale et physique, trad. d'Émile Cazelles, 1878 [1861], Paris, Germer Baillière, p. 83.

77. Plan, fo 47 , ms. gg 10, manuscrit conservé à la Bibliothèque municipale de Rouen, consultable sur le site Flaubert de l'université de Rouen, http://flaubert.univ-rouen.fr/ bouvard_et_pecuchet/ (consulté le 22 mai 2014). 
78. La synthèse flaubertienne, non dialectique, laisse du jeu entre les éléments. Il en découvre la formule en Orient et ses contrastes. Voir la lettre à Louise Colet du 27 mars 1853 ; Correspondance, II, op. cit., p. 283.

79. Cité par Béatrice Webb, My Apprenticeship, Londres, Cambridge University Press, 1979 [1926], p. 28, et repris dans Le Cas Spencer, p. 342. Elle a rendu une visite à Spencer en 1903.

INDEX

Mots-clés : Flaubert (Gustave), évolution, manuscrit, correspondance, Bouvard et Pécuchet

\section{AUTEUR}

\section{GISĖLE SÉGINGER}

Laboratoire LISAA, université Paris-Est Marne-la-Vallée et FMSH 